The following papers have been accepted for publication and will appear in 1979:

Herbert Amann and Peter Hess

A multiplicity result for a class of elliptic boundary value problems

A. Dzuraev

On a class of systems of singular integral equations on a bounded domain Alan R. Elcrat

Energy stability for the flow between rotating coaxial discs

George Havas, J. S. Richardson and Leon S. Stirling

The last of the Fibonacci groups

W. K. HaYman

On a non-regular parametric integral

D. B. Hinton and R. T. LEwIS

Singular differential operators with spectra discrete and bounded below

F. KAPPEL and W. SchappaCHER

Nonlinear functional differential equations and abstract integral equations

C. G. LeKKERKERKER

On eigendistributions in linear transport theory

A. C. McBride

Solution of dual integral equations of Titchmarsh type using generalized functions

J. D. P. Meldrum and A. Oswald

Near-rings of mappings

J. W. NeUberger

Boundary value problems for linear systems

V. A. NYE

Some comments on a singular elliptic problem associated with a rectangle Gordon Pagan and David Stocks

Oscillation criteria for second order hyperbolic initial value problems

A. Russell.

On certain fourth-order integral inequalities

Allan M. SinClair

Cohen elements in Banach algebras

GLENN WeBB

Compactness of bounded trajectories of dynamical systems in infinite dimensional spaces 


\section{(Section A)}

Volume 83

Parts 1/2

\section{CONTENTS}

S. B. HADID

Estimates of Liouville-Green type for solutions of systems of differential equations

S. D. COHEN

Composite rational functions which are powers

Guy Bolllat and Tommaso Ruggeri

Reflection and transmission of discontinuity waves through a shock wave. General theory including also the case of characteristic shocks

URI ELIAS

A classification of the solutions of a differential equation according to their asymptotic behaviour

MAN KAM KWONG

A second order Dirichlet differential expression that is not bounded below

STEVEN B. BANK

On the hypertranscendence of meromorphic solutions of certain functional equations

C. H. Houghton

Anti-isomorphisms of completely $\mathrm{O}$-simple semigroups

RUSSELL A. SMITH

The Poincare-Bendixson theorem for certain differential equations of higher order

JÜRGEN WEYER

Regularität in der nichtlinearen Spektraltheorie

J. M. HrLl and R. V. NiLlsen

Mutually conjugate solutions of formally self-adjoint differential equations

DOMENICO FUSCO

An asymptotic method for non-linear magnetosonic waves in an isothermal plasma with a finite conductivity

E. T. COPSON

Two series inequalities

L. E. Fraenkel

On regularized distance and related functions

C. R. Putnam

Hyponormal and essentially normal operators

PAOLO Nistri

Positive solutions of a non-linear eigenvalue problem with discontinuous non-linearity

BERND SCHULTZE

Green's matrix and the formula of Titchmarsh-Kodaira for singular left-definite canonical eigenvalue problems 